\section{Reflexiones teóricas sobre La estructura de capital de las pymes ${ }^{1}$}

Theoretical reflections on capital structure of SMEs

\author{
Gloria Mercedes Manrique Joya* \\ Gustavo Enrique Salazar Otálora**
}

Recibido: 14-08-2014 / Revisado: 10-09-2014 / Aceptado: 11-05-2015

\section{Abstract}

This article addresses theoretical background on the structure of capital cost of SMEs, with emphasis on a reflection on the theories developed by Modigliani and Miller (1958): the Theory MM (trade-off theory), Pecking Order Theory of Myers and Majluf (1984). It is also presented empirical referents to explain the financial structure and its resulting cost of capital, and finally an approach is made to the capital structure of SMEs in Colombia and the region under the model proposed by Berger and Udell (1998).

Keywords: capital cost, WACC weighted average cost of capital, capital structure, financial leverage, expected return, risk analysis. 


\section{Citación}

Manrique, G., Salazar, G. (2015). Reflexiones teóricas sobre la estructura de capital de las PYMES. Visión Empresarial, 1 (1), 12-29.

\section{Introducción}

La estructura de capital representa para las pequeñas y medianas empresas un cuello de botella, pues sus fuentes de financiamiento son reducidas, debido a su tamaño, tiempo de permanencia en el mercado, información financiera que poseen y concentración de la propiedad. Situación que refleja problemas por la asimetría de la información, riesgo por selección adversa y peligro moral; variables que fueron tratadas en las diferentes teorías desarrolladas en la materia y que se mencionan especialmente en Modigliani y Miller (1958; citados por Miller \& Modigliani, 1963): la teoría MM (la teoría trade-off) y la teoría de la jerarquía financiera (pecking order theory) de Myers y Majluf (1984), y de forma especial se estudia el modelo de Berger y Udell (1998), dada su especificidad y similitud con el comportamiento de las pymes en el entorno.

El artículo está estructurado de la siguiente manera: en la parte inicial se exponen algunos de los principales referentes teóricos inherentes a la valoración del costo de capital, y se presenta la estructura del capital y su relevancia dentro de la empresa.

En la segunda parte se realiza un acercamiento a los estudios empíricos desarrollados con base en las pequeñas y medianas empresas, tanto a nivel internacional, como a nivel nacional, para evidenciar cuáles han sido los resultados obtenidos, a fin de referenciar el comportamiento de las estructuras de capital, tanto en sectores, como en el tiempo.

En la tercera parte se hace una caracterización de las pymes en el contexto nacional y regional y se analiza el modelo de Berger y Udell (1998), dada la simplicidad del mismo y su ajuste a la composición de las pymes en un contexto general. 


\section{Referentes teóricos}

En la primera mitad del siglo XX, las finanzas se concebían bajo la idea de una concentración en ventas que representara una utilidad creciente y positiva dentro de la empresa, predominaba especialmente la planeación financiera de largo plazo, y su impacto en la adquisición de valor se aislaba de la evaluación de la empresa.

A partir de la mitad del siglo pasado se ha discutido sobre el acercamiento a la estructura óptima de capital, tanto en grandes, como en medianas y pequeñas empresas. El surgimiento de múltiples aspectos y consideraciones teóricas y empíricas, como los aportes de Modigliani y Miller (1958; citados por Miller \& Modigliani, 1963), que han transformado el mundo financiero y en especial el análisis del costo de capital en las compañías, significó una contradicción con las consideraciones clásicas o tradicionales, al suponer la aparición de un mercado de capitales perfecto.

Berle y Means (1932), con la teoría de los costos de agencia, y el problema de la separación entre la propiedad y el control o la administración, mostraron una relación directa entre el tamaño de la empresa, el cual puede prevalecer por el interés de la administración para incrementar el tamaño de la compañía por encima de los objetivos de maximización de utilidades. Esta teoría es retomada posteriormente por Franco, López y Muñoz (2009), quienes precisan que los conflictos de interés de los administradores provocan ineficiencias de gestión y asignaciones inadecuadas de recursos, generando costos ajenos a los intereses de los accionistas; y por Salas (1996, 2000), quien define el problema de agenciamiento, como un problema de asimetrías de información, pues el agente está mejor informado, posee datos especializados, mientras que el propietario no siempre tiene la misma condición de información, lo que genera conflictos de interés en la toma de decisiones, en muchos casos relacionados con el tema de estructura financiera (deuda).

Además aparecieron los problemas de agencia, que constituyeron un paradigma de la investigación sobre las finanzas corporativas durante los ochenta y de los cuales se ocuparon Jensen y Meckling (1976), quienes propusieron una regla para el accionista minoritario, y Shleifer y Vishny (1997), con iniciativas sobre el manejo de los mercados financieros, con el fin de establecer códigos de buen manejo corporativo.

Con Markowitz (citado por Martínez \& Restrepo, 2006) se polariza en los años sesenta el enfoque hacia el análisis del riesgo, en complemento con Sharper y Linner (citados por Martínez \& Restrepo, 2006), quienes hacen énfasis en el Capital Asset Pricing Model -CAPM- en una conjugación directa de la valoración del costo de capital con el riesgo sistemático, con base en las teorías desarrolladas por Markowitz en 1952. Este modelo propone la diversificación de carteras o portafolios de inversión, y 
estima el comportamiento de un inversionista adverso al riesgo -cuya intención al final de un período es maximizar su beneficio-, trabaja supuestos básicos como expectativas homogéneas por parte del inversor, y la existencia en el mercado de cantidades de activos con riesgo y activos libres de riesgo limitados (Martínez \& Restrepo, 2006).

Por otra parte, el WACC (weighted average cost of capital, o promedio ponderado) se refiere al costo sobre los recursos permanentes que requiere la empresa (pasivos LP, capital contable). Es importante para el accionista medir el costo de deuda, al igual que el costo por el aporte de sus recursos, el cual no puede ser inferior al costo de oportunidad del mercado, ya que no solo corre el riesgo de un deterioro patrimonial, sino también de una iliquidez que lleve a la desaparición de su negocio. Teóricamente el modelo expresa que entre más apalancamiento exista, es decir, cuanto mayor sea el aporte del socio, el costo de capital tiende a disminuir. En términos generales se identificará que la estructura financiera de la compañía debe optimizarse para ser más rentable, ya que el riesgo se aumenta en la medida en que quien asume la inversión es el propietario de la firma.

\section{La estructura de capital y su relevancia dentro de la empresa}

Las teorías sobre decisiones financieras más relevantes, y, a su vez, más conocidas, que han sido objeto de aplicaciones en el contexto nacional e internacional, aparecen en los años cincuenta con: Modigliani y Mi-

\section{Iler (1958): La teoría MM (la teoría trade-off)}

En su primera versión en 1958 (citados por Alarcón et al., 2007), esta teoría se estructuró sobre las siguientes premisas:

El costo de capital y el valor real de la empresa son totalmente independientes, es decir, el valor de los activos reales de la compañía no estaría influenciado por el valor de la deuda de la misma y por ende no habría afectación patrimonial.

Existe interrelación entre deuda y apalancamiento, teniendo en cuenta que la deuda, se supone, está libre de riesgo, entre más grande sea el aporte del socio, menor será la deuda y, por tanto, si el apalancamiento viene dado por deuda o emisión de acciones en circulación, la rentabilidad de esta tiende a disminuir.

De acuerdo con la estructura de endeudamiento de las empresas, la tasa interna de retorno (TIR) debe estar por encima del costo medio de capital, con el fin de que solo se efectúen aquellas inversiones que maximicen la rentabilidad del accionista o del propietario (medición de costo-beneficio).

El modelo de Modigliani y Miller (1958; citados por Alarcón et al., 2007), es desarrollado en condiciones 
de mercados perfectos, lo que hace que desconozca el impacto de los costes de quiebra, puesto que se asume que esta se da de manera inmediata, se excluyen los impuestos y la compensación fiscal que se deriva de los mismos por el pago de intereses, al igual que se omite la incertidumbre. Para Brealey, Myers y Allen (2006), el modelo de Modigliani y Miller (1958), aparte de las anteriores falencias, también presenta conflicto de intereses de los propietarios, sobre el incentivo que puede generar la deuda sobre las inversiones y los dividendos.

Este modelo fue replanteado en 1963 por los mismos autores, incluyendo el impacto de los impuestos sobre la rentabilidad neta de la compañía. En muchas ocasiones los beneficios fiscales atenúan la deuda e indirectamente actúan en la estructura óptima de capital y la rentabilidad financiera; también se tomaron en cuenta los costos de quiebra y otro tipo de beneficios por impuestos dada la relación costo-beneficio.

La teoría trade-off deriva un equilibrio estático, a partir de los costos de quiebra y los beneficios generados en los impuestos y por el pago de intereses de deuda; también ofrece una estructura óptima de capital que minimiza los costos y maximiza el valor en la empresa. Con esta teoría se admite la presencia de un índice de endeudamiento óptimo dentro de lasempresas, (Taggart Jr., 1977; Marsh, 1982; Jalilvand \& Harris, 1984; citados por Ferrer \& Tresierra, 2009, p. 67), lo que hace predecir que empresas con altos activos tangibles pri- vilegien el endeudamiento para beneficiarse en costos por la compensación fiscal, y, por el contrario, empresas con activos riesgosos e intangibles puedan estar pensando en optar por un endeudamiento propio (Brealey, Myers \& Allen, 2006). Aunque, sin lugar a dudas, esta teoría ha logrado precisar importantes aspectos con relación a una estructura adecuada de capital en las empresas, aún existen vacíos dentro de la misma que no permiten explicar por qué a veces las empresas con un monto de activos tangibles importantes de respaldo a deuda, presentan índices de endeudamiento bajos.

\section{La asimetría de la información y la teoría de la jerar- quía financiera (pecking order theory) desarrollada por Myers y Majluf (1984)}

El dilema aquí es que no se conoce con certeza una óptima estructura de capital. Se sobreestima el valor presente de las inversiones y no se valora adecuadamente el capital de la deuda.

En su orden de prioridad, las empresas para financiarse agotan los recursos internos, recursos por los cuales el accionista fijará una tasa de rentabilidad esperada; luego acuden a la deuda externa y por último a las acciones. La finalidad del modelo es la maximización de la rentabilidad del accionista a partir del análisis de la jerarquización de los recursos. Una vez agotados los recursos internos, deben identificar en qué medida se recurre a los recursos externos. El proceso de jerarquización se da 
en función del comparativo entre el flujo de caja interno y el déficit financiero, considerando un orden prelacional:

- Financiamientos internos, basados en la apropiación de utilidades.

- Prelación de un bajo riesgo en el endeudamiento.

- Emisión accionaria.

Un aspecto importante de esta teoría es la asimetría con que se manejan las decisiones, pues se asume que el gerente tiene mayor información interna de la compañía que los accionistas externos o nuevos, la cual va a manejar a favor de los actuales accionistas. En el caso de empresas cotizantes en bolsa, esta información servirá para tomar decisiones en favor de uno u otro, en relación con el capital accionario (selección adversa, riesgo moral).

A diferencia de la anterior teoría, en esta el índice de endeudamiento no es estático ni está asociado al valor de la empresa, este varía de acuerdo con los cambios en los flujos de caja internos, los dividendos netos y las inversiones. Empresas muy rentables con altas oportunidades de inversión mantienen niveles de endeudamiento bajo porque no lo requieren, la jerarquización de deuda y su capacidad le permiten acudir a recursos autogenerados o propios, y, a su vez, empresas menos rentables se endeudan más, porque sus recursos internos no son suficientes para cubrir sus necesidades de inversión (Fama \& Kenneth, 2002). El modelo de Myers y Majluf se ha extendido en varias direcciones.
Por ejemplo, Krasher (1986, citado por Tenjo et al., 2007) ha estudiado el caso en donde el tamaño de la inversión es una variable de elección. Heinkel y Zecher (1990, citados por Tenjo et al., 2007), al igual que Narayanan (1988, citado por Tenjo et al., 2007), han obtenido resultados similares a los de Myers y Majluf en un contexto donde la asimetría de información se encuentra únicamente sobre la nueva inversión.

\section{Un acercamiento a la estructura de capital de las empresas, evidencia empírica}

Varios estudios empíricos, tanto en el contexto internacional como nacional, han tratado de contrarrestar las teorías expuestas, aunque han llegado a resultados diversos. En la Tabla 1 se observan algunos referentes sobre trabajos elaborados en torno a la estructura de capital de las empresas.

Desde la perspectiva del trade-off, el equilibrio estático, en lo contextualizado por Aghion y Bolton (1991-1992) y Michaelas, Chittenden y Poutziouris (1999), las pymes tienen tasas de rentabilidad muy bajas, por lo que se enfrentan a tasas fiscales marginales de impuesto muy bajas, y niveles de impuestos por deducir por un monto pequeño, mientras que el riesgo de quiebra es elevado, lo que implica que ante unos beneficios fiscales bajos y un alto costo por exposición a la quiebra, las pequeñas empresas no privilegien la deuda. Y de acuerdo con Rivera (2002), las empresas con mayores activos privilegian el endeuda- 
Tabla 1. Estudios empíricos sobre estructuras de capital

\begin{tabular}{|c|c|}
\hline $\begin{array}{l}\text { Estudios empíricos a } \\
\text { nivel internacional }\end{array}$ & $\begin{array}{l}\text { Estudios empíricos a } \\
\text { nivel nacional }\end{array}$ \\
\hline Ang y Jung & $\begin{array}{l}\text { Barahona y Gómez (2010). Aspectos conceptuales y } \\
\text { empíricos de la financiación de las nuevas empresas en Colombia. }\end{array}$ \\
\hline $\begin{array}{l}\text { Berger y Udell, } \\
1998\end{array}$ & $\begin{array}{l}\text { Buenaventura y Arismendi (2006, Septiembre). Las } \\
\text { teorías de financiación empresarial y las pymes: estudio del } \\
\text { pecking order en el sector de confecciones de Colombia. }\end{array}$ \\
\hline & $\begin{array}{c}\text { Burbano et al. C (2011). Modelo de estructura } \\
\text { financiera para las pymes del sector metalmecánico } \\
\text { del corredor industrial de Boyacá. }\end{array}$ \\
\hline $\begin{array}{l}\text { Holmes y Kent, } \\
1991\end{array}$ & $\begin{array}{l}\text { Cruz (2012). Medición y análisis de un modelo para la } \\
\text { determinación del costo de capital de las pymes } \\
\text { del subsector de confección de Bogotá. }\end{array}$ \\
\hline $\begin{array}{l}\text { Michaelas, } \\
1999\end{array}$ & $\begin{array}{l}\text { Gama (2009). Costo de capital: estudio de la incidencia de } \\
\text { las variables determinantes del costo de capital y el consi- } \\
\text { guiente reflejo en los estado financieros de las pymes del } \\
\text { sector avícola de Bogotá, durante el período 2000-2005. }\end{array}$ \\
\hline $\begin{array}{l}\text { Petersen y Rajan, } \\
1997\end{array}$ & $\begin{array}{l}\text { Rivera (2008). Decisiones de financiación de la } \\
\text { industria metalmecánica en el Valle del Cauca. }\end{array}$ \\
\hline & $\begin{array}{l}\text { Rivera (2007). Estructura financiera y factores determi- } \\
\text { nantes de la estructura de capital de las pymes del sector } \\
\text { confecciones del Valle del Cauca del período 2000-2004. }\end{array}$ \\
\hline Sánchez-Vidal y & \\
\hline Matín-Ugedo, 2005 & $\begin{array}{l}\text { Rivera, J (1998). Factores determinantes de la estructura de } \\
\text { capital de las grandes empresas industriales de Colombia. }\end{array}$ \\
\hline $\begin{array}{l}\text { Watson y Wilson, } \\
\qquad 2002\end{array}$ & $\begin{array}{l}\text { Tenjo et al. (2006). Determinantes de la estructura de } \\
\text { capital de las grandes empresas de Colombia. }\end{array}$ \\
\hline
\end{tabular}

Fuente: elaboración de los autores con base en Otero, Fernández \& Vivel (s.f.). miento, dada su capacidad de garantía frente al mismo.

Así mismo, frente a la teoría de la jerarquía financiera (pecking order), que puede verse mejor explicada en Watson y Wilson (2002), la prioridad del accionista actual por mantener la propiedad y el control sobre la empresa condicionan la fuentes de financiamiento (citados por Holmes \& Kent, 1991), yuxtaponiendo la emisión accionaria por la deuda. De igual forma en esta teoría la asimetría de la información conduce a ocultar información, lo que implica para el nuevo accionista un riesgo de selección adversa y un perjuicio tipificado en el riesgo moral.

\section{Según Sánchez-Vidal y Mar-} tín-Uguedo (2005), las pymes en países no anglosajones presentan una dificultad de acceso a los mercados de capitales, lo que hace que recurran de manera permanente a la autogeneración de recursos o la deuda externa, implicando con ello una jerarquización similar a la expuesta por Myers y Majluf (1984), 
por externalidades del mercado de recursos a la hora de definir su estructura de capital. Teoría que se refuerza en Berger y Udell, (1998), en cuanto la jerarquía de deuda, por falta de acceso a recursos externos como los mercados de capitales, ocasiona costos más elevados en la estructura de capital y trámites más dispendiosos, lo que hace que los gerentes tomen decisiones de corto plazo, sin tener en cuenta que las tasas pueden ser más elevadas y pueden inducir a un riego de quiebra.

En este mismo sentido, Berger y Udell (1998) proponen examinar la estructura de capital en concordancia con el ciclo de vida de las empresas, y Aghion y Bolton (1991-1992) establecen que es probable que los cambios en el tiempo de las organizaciones, obliguen a pensar que la estructura de capital no se da por equilibrio estático, ni existe una única teoría de explicación, por el contrario, en cada etapa la empresa puede adoptar un esquema teórico bajo su conveniencia. En igual dirección, Tenjo et al. (2006) y Tenjo y García (1996), en los estudios empíricos adelantados en el ámbito nacional, han encontrado importantes hallazgos, demostrando que la relación de la deuda de la mediana empresa es inversamente proporcional a la rentabilidad, y que existe una relación negativa con el crecimiento y la edad de la empresa en el mercado.

Por su parte, Rivera (1998), siguiendo la teoría del pecking-order, encuentra que las empresas en Colombia usan como principal fuente de financiamiento el endeudamiento de corto plazo, seguido de la retención de utilidades y luego la emisión accionaria; además, la actividad económica de las empresas no es un factor determinante, como si lo es el tamaño y el ciclo de vida de las mismas. Las grandes empresas se inclinan por la autofinanciación de recursos, la mediana por la deuda de corto plazo y las pequeñas por la búsqueda de un nuevo socio. Vale decir que es un factor limitante dentro de la teoría de la jerarquía en el mercado nacional, el poco acceso a recursos de capital (emisión de acciones).

En Buenaventura y Arizmendi (2006), se deduce una especial preferencia de las pymes de las confecciones del Valle del Cauca por la deuda de corto plazo, y se determina que existe una correspondencia negativa entre esta y la rentabilidad; en muchos casos las empresas usan recursos internos para financiar sus proyectos, dada la correlación positiva de este medio de apalancamiento y la rentabilidad (lo que refleja el uso de la teoría del pecking order). En relación con la teoría del trade-off, se encuentra que esta no se ajusta al sector, puesto que la hipótesis planteada en el estudio está en contravía con la teoría de la información asimétrica y los costos de agencia ante la subinversión.

Cruz, Villareal y Rosillo (2003) expresan variables coincidentes con las de Rivera (2004) en el sector de las confecciones en Bogotá. En este, las empresas privilegian el endeudamiento externo de largo plazo, por encima de los costosos recursos de los inversionistas 
particulares. Al igual que Rivera (2004), se concluye que el estudio puede estar influenciado por el tamaño de las empresas, lo que implica la existencia de la teoría de trade-off, al presentar una correlación positiva entre la rentabilidad y los recursos internos, más la utilidad, menos endeudamiento.

\section{Una aproximación a la estructura de capital de las pymes}

Históricamente, las pymes han pasado a ser elementos indispensables de la actividad económica, tanto en los países desarrollados como en los países en transición (ONU, 1997). En América Latina y en especial en Colombia, más del 90 \% de las empresas, de acuerdo con lo establecido en la Ley 590 del 10 de junio de 2000, se clasifica como pequeña y mediana empresa, y se resume en algunos de los siguientes aspectos:

Tabla 2. Características de las pymes en términos de personal-activos.

\begin{tabular}{|c|c|c|c|}
\hline & Mediana empresa & Pequeña empresa & Microempresa \\
\hline Personal & $\begin{array}{l}\text { Entre } 51 \text { y } 200 \\
\text { trabajadores }\end{array}$ & $\begin{array}{l}\text { Entre } 11 \text { y } 50 \\
\text { trabajadores }\end{array}$ & $\begin{array}{l}\text { Menor o igual a } \\
10 \text { trabajadores }\end{array}$ \\
\hline $\begin{array}{l}\text { Activos } \\
\text { totales }\end{array}$ & $\begin{array}{c}\text { De } 100.000 \text { a } 610.000 \\
\text { UVT= unidades } \\
\text { de valor tributario. } \\
\text { Ley } 1111 \text { de } 2006 .\end{array}$ & $\begin{array}{l}\text { Entre } 501 \text { y } \\
5000 \text { SMMLV }\end{array}$ & $\begin{array}{l}\text { Menor de } 500 \text { SMMLV, } \\
\text { excluida la vivienda. }\end{array}$ \\
\hline
\end{tabular}

Fuente: tomado de Pymesfuturo.com

De acuerdo con Aghion y Bolton (1991-1992), para que una empresa sea considerada pyme, debe poseer las siguientes características: no emitir valores negociables, los propietarios no deben poseer un portafolio de inversión diversificado, los dueños deben ser emprendedores, enfrentar altos costes de deuda y no contar con una organización gerencial completa.

En Colombia, y específicamente en el departamento de Boyacá, (al igual que en la mayoría de los países de América Latina), la estructura empre- sarial se fundamenta en las pequeñas empresas; en concordancia con la información presentada por el Departamento Nacional de Estadística (DANE) y Confecámaras, la distribución del sistema productivo nacional es la siguiente:

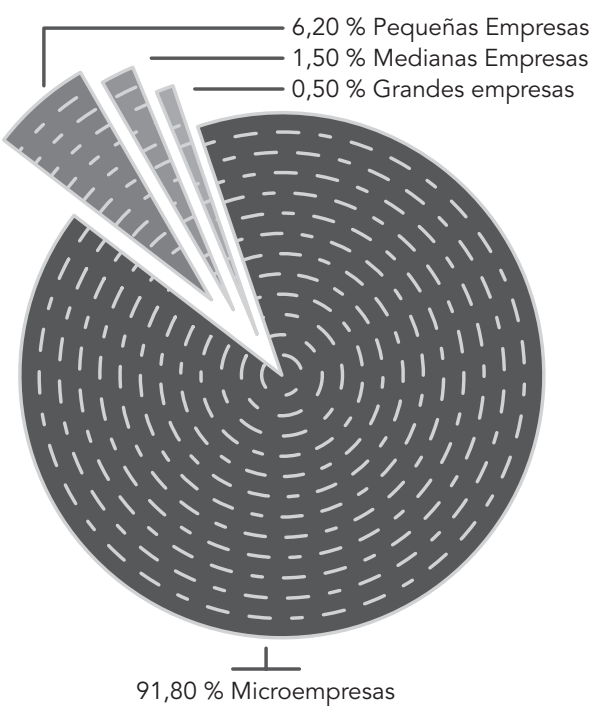

Gráfica 1. Distribución porcentual del nivel empresarial por tamaño en Colombia. Fuente: construcción de los autores con base en Información Dane, Confecámaras.

En el país, las pymes enfrentan problemas en el reflejo de su información financiera, lo que impide un acceso fácil a las fuentes de financiamiento, y en muchos casos por su constitución, la cual privilegia las sociedades limitadas y unipersonales, al igual que por su tamaño, le 
restan posibilidades de acceder al mercado de capitales.

Las pymes en su gran mayoría dependen de los recursos aportados por los propietarios, lo que eleva el riesgo de quiebra y la baja permanencia en el mercado; además, en los primeros años de operación existe poca compensación por inversión al propietario, tesis que se confirma con los datos de las Cámaras de Comercio de Boyacá: (ver Tabla 3).

Con estas singularidades en las pymes, y retomando variables como tamaño, propiedad, antigüedad, se aborda nuevamente el racionamiento expresado en párrafos anteriores, sobre el ciclo de vida de las empresas expuesto por Berger y Udell (1998; citados por Ferrer \& Tresierra, 2009, p. 76), quienes toman como supuesto de partida la evolución constante de las pymes en términos de tamaño, edad y disponibilidad de la información, los cuales actúan como determinantes de su estructura de capital.
Tabla 3. Estadística de disolución de sociedades en el departamento de Boyacá.

\begin{tabular}{|c|c|c|c|c|c|c|}
\hline \multicolumn{7}{|c|}{$\begin{array}{l}\text { Sociedades disueltas en Boyacá, según actividad } \\
\text { económica en el periodo 2008-2009 }\end{array}$} \\
\hline \multirow{3}{*}{$\begin{array}{l}\text { Actividad } \\
\text { económica }\end{array}$} & \multicolumn{5}{|c|}{ Año corrido } & \multirow{3}{*}{$\begin{array}{c}\text { Var. \% } \\
\text { Anual }\end{array}$} \\
\hline & \multicolumn{3}{|c|}{2008} & \multicolumn{2}{|c|}{2009} & \\
\hline & $\mathrm{N}^{\circ}$ & ! & Valor & $\mathrm{N}^{\circ}$ & Valor & \\
\hline $\begin{array}{l}\text { Agricultura, ganadería, caza, } \\
\text { silvicultura y pesca }\end{array}$ & 5 & $\begin{array}{l}1 \\
\vdots \\
1 \\
1\end{array}$ & 45.990 & 5 & 3.051 .017 & $6.534,1$ \\
\hline Explotación de minas y calderas & 10 & $\begin{array}{l}1 \\
1\end{array}$ & 398.615 & 5 & 1.860 .357 & 366,7 \\
\hline Industria manufacturera & 7 & $\begin{array}{c}1 \\
\vdots \\
1\end{array}$ & 621.170 & 10 & 408.684 & $-34,2$ \\
\hline Electricidad, gas y agua & 0 & $\begin{array}{c}1 \\
1 \\
1 \\
1\end{array}$ & 0 & 0 & : ton & (..) \\
\hline Construcción & 6 & $\begin{array}{l}1 \\
1 \\
1\end{array}$ & 3.615 .019 & 7 & 791.583 & $-78,1$ \\
\hline $\begin{array}{l}\text { Comercio y reparación } \\
\text { de vehículos }\end{array}$ & 43 & $\begin{array}{l}1 \\
1 \\
1\end{array}$ & 6.457 .744 & 27 & 7.368 .305 & 113,1 \\
\hline Hoteles y restaurantes & 2 & $\begin{array}{l}\text { ! } \\
\text { I }\end{array}$ & 33.930 & 2 & 62.000 & 82,7 \\
\hline $\begin{array}{l}\text { Transporte, almacenamiento } \\
\text { y comunicaciones }\end{array}$ & 12 & $\begin{array}{l}1 \\
1 \\
1 \\
1\end{array}$ & 508.934 & 9 & 2.697 .312 & 430,0 \\
\hline Intermediación financiera & 1 & $\begin{array}{l}1 \\
\vdots \\
1\end{array}$ & 20.000 & 3 & 4.500 & $-77,5$ \\
\hline $\begin{array}{l}\text { Actividades inmobiliarias, } \\
\text { empresariales y de alquiler }\end{array}$ & 14 & $\begin{array}{l}1 \\
\vdots \\
1 \\
1\end{array}$ & 121.825 & 18 & 1.114 .646 & 815,0 \\
\hline Educación & 1 & $\begin{array}{c}\vdots \\
\vdots \\
1\end{array}$ & 1.000 & 2 & 232.166 & (..) \\
\hline Servicios sociales y de salud & 7 & $\begin{array}{l}1 \\
1 \\
1\end{array}$ & 144.079 & 6 & 2.716 .927 & 1.785 \\
\hline $\begin{array}{l}\text { Otros servicios, comunitarios, } \\
\text { sociales y personales }\end{array}$ & 4 & $\begin{array}{l}1 \\
1 \\
1 \\
1\end{array}$ & 784.733 & 5 & 655.995 & $-16,4$ \\
\hline Total & 112 & 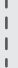 & 9.753 .039 & 99 & 20.963 .492 & 114,9 \\
\hline
\end{tabular}

Fuente: Cámaras de Comercio de Tunja, Duitama y Sogamoso 
Las pymes, en la medida en que crecen y adquieren experiencia en el mercado, cambian sus fuentes de financiamiento. Gráfica 2:

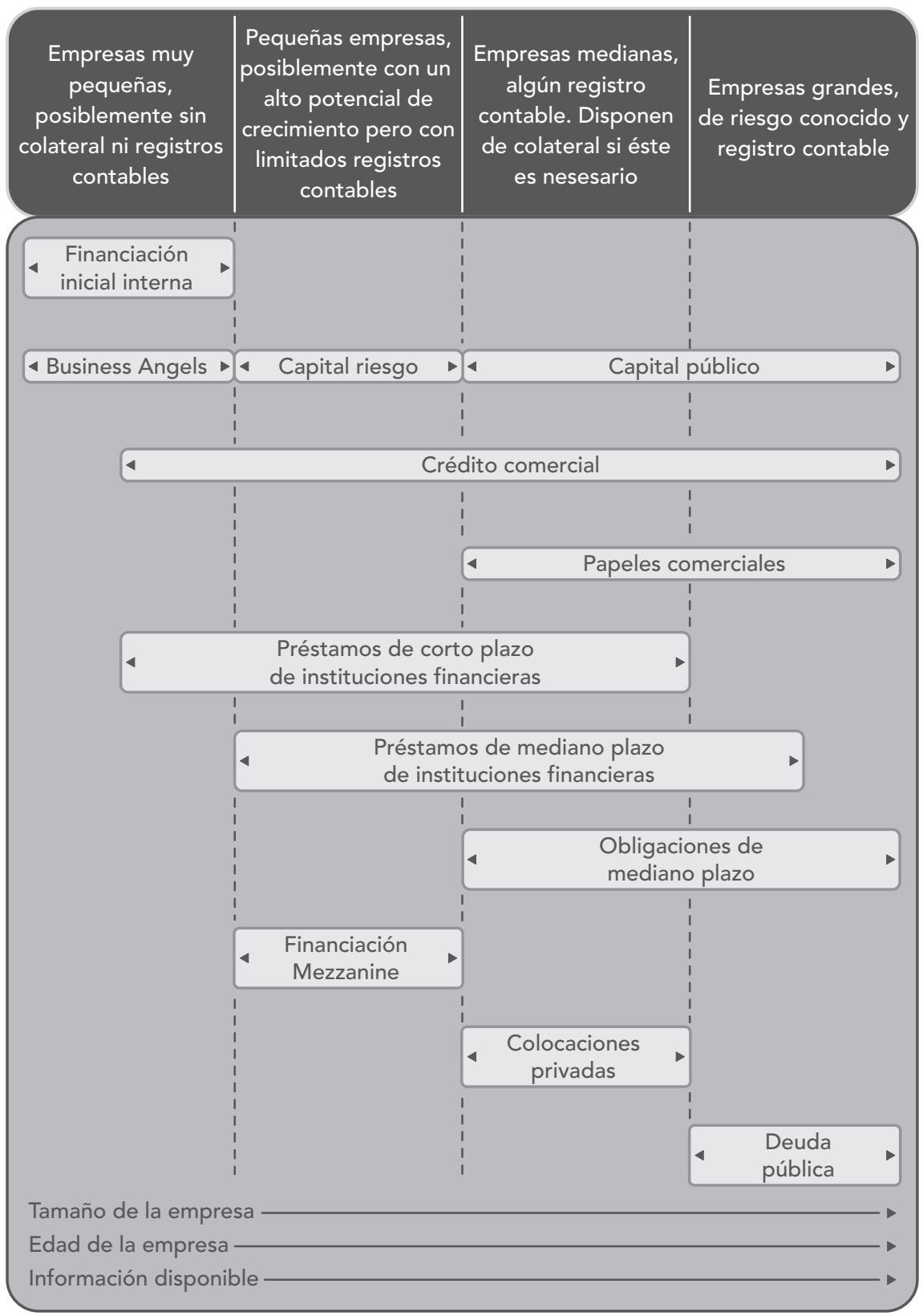

Gráfica 2. El ciclo de crecimiento financiero de las empresas. Fuente: tomado de Ferrer \& Teseirra (2009).
En lo propuesto por Berger y Udell (1998), se destaca que las empresas tanto en el contexto nacional como en el regional tienen un comportamiento muy cercano al del ciclo de vida: en su etapa inicial, recurren a la financiación interna, al crédito comercial o a los business angels ${ }^{2}$; a medida que se fortalecen o aumentan su tamaño acuden a capital de riesgo (accionistas minoritarios), y a fuentes de financiamiento bancario, y a medida que cambia su composición o aumenta su tamaño, pueden ampliar sus posibilidades de financiación a mercados más especializados 3 .

De acuerdo con Berger y Udell (1998), la secuencia en que las pymes empiezan a obtener recursos, agotadas las fuentes internas, según las teorías del trade-off y del pecking

\footnotetext{
2 Los business angels son individuos que invierten directamente en empresas pequeñas, generalmente mediante acciones comunes (Berger \& Udell, 1998).

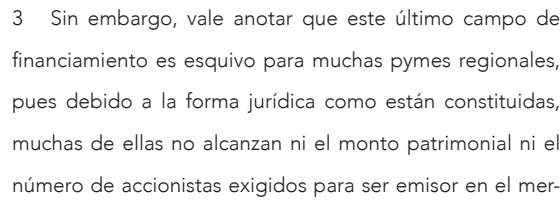


order, son las fuentes externas; comportamiento que se refuerza con los datos arrojados por la gran encuesta para pymes 2013, aplicada por el Centro de Estudios Económicos de la Asociación Nacional de Instituciones Financieras (ANIF):

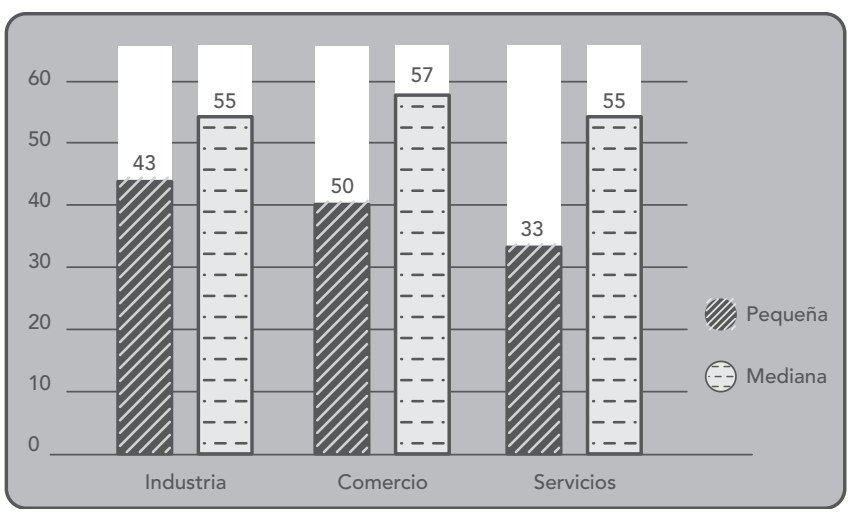

Gráfica 3. Respuestas afirmativas a las solicitudes de crédito realizadas en el 2012.

Fuente: ANIF (2013) y Asobancaria (2010).

En Colombia las fuentes de financiamiento bancario son las más utilizadas por las pymes, debido a la política de profundización financiera iniciada en los últimos años y a la masificación de recursos de colocación de las entidades financieras. El mercado presenta entre un 47 y $54 \%$ de solicitudes de crédito por parte de las pymes, aunque en el último año se presentó un descenso en el porcentaje de solicitudes aprobadas en la pequeña empresa, en las medianas se sostiene y se da un porcentaje de crecimiento en el sector comercio. Existen evidencias para corroborar que este tipo de apalancamiento es el más usado por los propietarios a pesar de que se reconoce en él un alto costo por uso.
Se privilegian otras fuentes como business angels, aunque quedan expuestos a una selección adversa o a riesgo moral, en relación con las asimetrías de la información, además es una fuente a la que los propietarios acuden si el nuevo inversor tiene algún tipo de relación familiar o de amistad, para evitar la desconcentración de poder.

\section{Conclusiones}

Las teorías sobre decisiones financieras más importantes, conocidas y aplicadas en el contexto regional nacional e internacional, aparecen en los años cincuenta con Modligiani y Miller, la teoría MM (trade off), la cual considera que el costo de capital y el valor real de la compañía son independientes, es decir, el valor de los activos reales no está influenciado por la deuda. De acuerdo con la estructura de endeudamiento de las empresas, la tasa interna de retorno (TIR) debe estar por encima del costo medio de capital, con el fin de que solo se efectúen las inversiones que maximicen la rentabilidad del propietario. El modelo presentado por los autores citados, es desarrollado en condiciones de mercados perfectos, lo que hace que se desconozca el impacto de los costes de quiebra, los impuestos y la compensación fiscal que se deriva de los mismos por el pago de intereses, al igual que la incertidumbre.

Por dicha razón, este modelo fue replanteado por los mismos autores en 1963, incluyendo el impacto de los 
impuestos sobre la rentabilidad neta de la compañía, puesto que en muchas ocasiones los beneficios fiscales atenúan la deuda e indirectamente actúan en la estructura óptima del capital y la rentabilidad financiera; también tomaron en cuenta los costos de quiebra y otro tipo de beneficios por impuestos, dada la relación costo-beneficio, haciendo más completo dicho modelo.

En contexto, la evidencia deja al descubierto que en casos como las pequeñas empresas, el retorno de la inversión está por debajo del costo de capital asumido, dado el tamaño del mercado, las escazas fuentes de financiamiento y la necesidad de sobrevivencia de las mismas, que las obligan a operar en condiciones no óptimas de rentabilidad financiera; y que además, analizando lo propuesto por Brealey, Myers y Allen (2006), la deuda en muchas compañías pequeñas no es un atenuante fiscal, cuando se opera con márgenes de rentabilidad tan estrechos, que no sustituyen ni siquiera el gasto financiero, lo que conlleva una pérdida del ejercicio, trasladando la instancia fiscal a otros escenarios donde la tributación se vuelve un costo patrimonial.

Bajo la teoría de la jerarquía financiera (pecking order theory), de acuerdo con la normatividad y el modelo de constitución empresarial existente en Colombia y extendido a las regiones, es difícil que se dé un análisis óptimo de la jerarquización de los recursos; empresas muy rentables con altas oportunidades de inversión mantienen niveles de endeudamiento bajo, porque no lo requieren, su capacidad les permite acudir a recursos propios; pero, a su vez, empresas menos rentables se endeudan más, porque sus recursos internos no son suficientes para cubrir sus necesidades de inversión, y ante el riesgo que representa para muchas instituciones financieras la baja rentabilidad y la escaza consolidación de la misma años tras año, estos compensan el mismo a través de mayores tasas de colocación de recursos, elevando el costo de capital de las pymes en contravía de una tasa de retorno apropiada para el esfuerzo de inversión y de gestión que realiza el empresario.

De igual forma, bajo el análisis de las teorías modernas sobre estructura de capital, teoría del equilibrio estático de la estructura de capital y de la jerarquía financiera, el escenario de análisis es un poco más complejo, puesto que se toman como marco de referencia grandes empresas y mercados de capitales desarrollados, por lo que en determinados escenarios hacen difícil la medición de los costes de capital en pequeñas empresas. Además, en estas teorías, la asimetría de la información en las pymes se acentúa mucho más, ya que estas no poseen información financiera completa, y su concentración de poder y manejo obstaculiza el proceso de inyección de recursos.

Sin embargo, es el modelo del ciclo de crecimiento financiero propuesto por Berger y Udell (1998), el que parece ajustarse más al comportamiento de las pymes, dado que se espera que con el paso del tiempo se 
dé una mutación de empresas pequeñas a grandes empresas (o las pequeñas empresas se vuelvan ordenadas en la presentación de su información financiera), lo que corregiría los problemas de asimetría de la información, disminuiría los riesgos de quiebra, peligro moral y selección adversa y, a su vez, permitiría ampliar las posibilidades de conseguir recursos externos, facilitando el modelo de jerarquización (Aghion \& Bolton, 1991-1992), porque en cada etapa en las empresas puede ser explicado por un determinado modelo de estructura de capital.

A nivel general, se puede deducir que aunque hay dificultades en el acercamiento óptimo a la estructura de capital, debido a que las pymes a nivel nacional y regional presentan muchos problemas a la hora de reflejar su información financiera, existe una base teórica fuerte para su análisis. La investigación teórica expuesta refleja una convergencia en cuanto a la búsqueda de la optimización financiera de las empresas, que sirve como referente para las pequeñas y medianas empresas. Igualmente, se puede inferir que de acuerdo con el tamaño y la edad de las empresas existen beneficios tributarios para el apalancamiento financiero externo, los cuales, en muchos casos, no son aprovechados al máximo por las pymes, dada la baja cultura financiera. Finalmente, son puntos para abordar en la estructura de capital de las pymes: la dependencia de los recursos aportados por los propietarios, lo que eleva el riesgo de quiebra y la baja permanencia en el mercado; además, en los primeros años de operación existe poca compensación por inversión al propietario. De acuerdo con lo expuesto por Sánchez-Vidal y Martín-Uguedo, (2005), se puede inferir que en Colombia, al igual que en otros países de América Latina, la mayoría de las pymes presenta una dificultad de acceso a los mercados de capitales, lo que hace que recurran de manera permanente a la autogeneración de recursos o a la deuda externa, especialmente a la deuda financiera, sin una planeación adecuada frente a los retornos de capital y la rentabilidad esperada. 


\section{Referencias}

Aghion, P. \& Bolton, P. (1991-1992). An incomplete contracts approach to financial contracting. Review of Economic Studies Limited, 59.

Alarcón, A. et al. (2007, enero). La teoría sobre estructura financiera en empresas mixtas hoteleras cubanas. Observatorio de la Economía Latinoamericana, (73).

ANIF (2013). Gran encuesta para pymes 2013. Bogotá: ANIF.

Asobancaria. (2010). Las pymes y la importancia en el crecimiento económico. Bogotá: Asobancaria.

Berle, A. \& Means, G. (1932). The modern corporation and private property. Nueva York: MacMillan.

Berger, A. N. \& Udell, G.F. (1998). The economics of small business finance: The roles of private equity and debt markets in the financial growth cycle. Journal of Banking \& Finance, 22(6-8), 613-673.

Brealey, R., Myers, S. \& Allen, F. (2006). Principios de finanzas corporativas. (8 Ed.). Madrid: McGraw-Hill.

Cruz, S., Villareal, J. \& Rosillo, J. (2003). Finanzas corporativas. Valoración, política de financiamiento y riesgo. Teoría \& Práctica. Bogotá: Thomson.

Fama, E. \& Kenneth, R. (2002). Testing trade-off and pecking order predictions about dividends and debt. The Review of Financial Studies, 15(1) 1-33. 
Ferrer, M.A. \& Tresierra, A. (2009). Las pymes y las teorías modernas sobre estructura de capital. Red de Revistas Científicas de América Latina y el Caribe, España y Portugal Sistema de Información Científica, Redalcy.org. Recuperado de http://www.redalyc.org/articulo.oa?idp=1\&i$d=88012314007 \& c i d=51984$.

Franco, G., López, L. \& Muñoz, G. (2009). Determinantes de la estructura de capital de las grandes empresas manufactureras en Uruguay. Trabajo final de la Maestría en Finanzas. Udelar, Uruguay.

Holmes, S. \& Kent, P. (1991). An empirical analysis of the financial structure of the small and large Australian manufacturing enterprises. Journal of Small Business Finance, 1 (2), 141-154.

Jensen, M. C. \& Meckling, W. (1976). Theory of the firm: managerial behavior. Agency Costs and Ownership Economics, 3.

Martínez, C. L. \& Restrepo, J. A. (2006). Selección de portafolios usando simulación y optimización bajo incertidumbre. S.I.: Red Dyna.

Michaelas, N., Chittenden, F. \& Poutziouris, P. (1999). Financial policy and capital structure choice in U.K. SMEs: empirical evidence from company Panel Data. Small Business Economics, 12 (2), 113-130.

Miller, M. \& Modigliani, F. (1963). Corporate income taxes and the cost of capital: a correction. The American Economic Review. 
Myers, S. C. \& Majluf, N. S. (1984). Corporate financing and investment decisions when firms have information that investors do not have. Journal of Financial Economics 13 (2), 187-221. doi:10.1016/0304405X (84)90023-0

ONU (1997, 13 de mayo). Conferencia de Naciones Unidas sobre Comercio y Desarrollo. ID/B/COM.3/EM.2/2.

Rivera, J. (1998). Factores determinantes de la estructura de capital de las grandes empresas industriales en Colombia. Tesis doctoral presentada a la Universidad Autónoma de Madrid.

Rivera, J. (2002). Teoría sobre la estructura de capital. Estudios Gerenciales.

Rivera, J. (2004). Estructura financiera y factores determinantes de la estructura de capital de las pymes del sector de confecciones del Valle del Cauca en el período 2000-2004. Cuadernos de Administración, 20, (34), 191- 219.

Salas, V. (1996). Economía de la empresa: decisiones y organización. Barcelona: Ariel Economía.

Salas, V. (2002). El gobierno de la empresa: presentación. Ekonomiaz, (50), 10-27.

Sánchez-Vidal, J. \& Martín-Ugedo, J. (2005). Financing preferences of spanish firms: evidence on the pecking order theory. Review of Quantitative Finance and Accounting, 25 (4), 341-355. 
Shleifer, A. \& Vishny, R. W. (1997). A survey of corporate governance. The Journal of Finance, (52).

Tenjo, F. et al. (2007). Los ciclos de la inversión y su financiamiento en Colombia. En Borradores de Economía, n. 438. Bogotá: Banco de la República, Colombia.

Tenjo, F. \& García, G. (1996). Desarrollo financiero y estructura de capital de las empresas. Banca y Finanzas (40), 5-34. 